\title{
Supporting Information: Analytic Device Model of Organic Field-Effect Transistors with Doped Channels
}

Shiyi Liu, ${ }^{*}$ Raj Kishen Radha Krishnan, ${ }^{*}$ Drona Dahal, ${ }^{*}$ and Björn Lüssem* Department of Physics, Kent State University, Kent, OH, 44240

E-mail: sliu20@kent.edu; rradhakr@kent.edu; ddahal2@kent.edu; blussem@kent.edu 


\section{$1 \quad$ Stability of intrinsic OFETs}

(a)

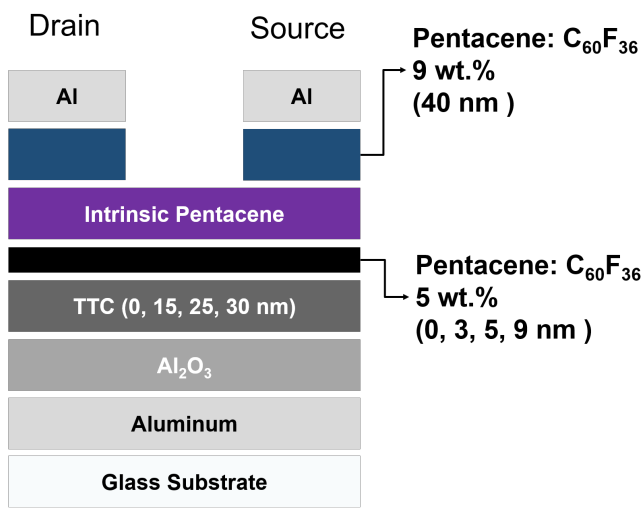

(b)

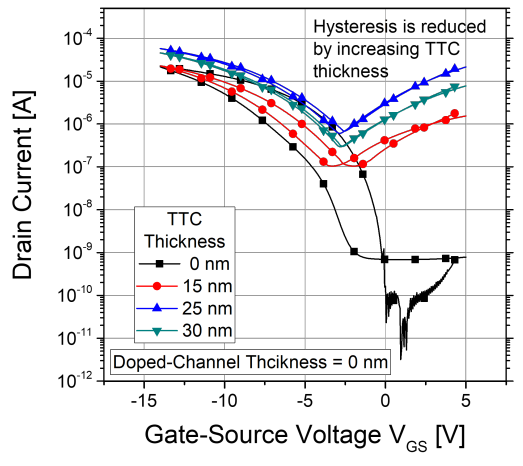

Figure S1: (a) Schematic structure of doped OFETs with varying thickness of the TTC and the doped channel. The doped channel is inserted between the passivation layer (TTC) and the intrinsic Pentacene, which is a thin film of p-doped Pentacene ( $5 w t$.\% Pentacene: $\left.C_{60} F_{36}\right)$. (b) Transfer characteristics of intrinsic OFETs for different thickness of TTC. The thickness of TTC is varied from 0 to $30 \mathrm{~nm}$ to optimize the performance of intrinsic OFETs as shown in (a). The intrinsic Pentacene OFETs switch from an unipolar (p-type only) to an ambipolar (p- and n-type) characteristic with increasing the thickness of TTC, which suppresses the traps at the surface of the gate oxide $\left(\mathrm{Al}_{2} \mathrm{O}_{3}\right)$.

In order to verify the model discussed in the main text, OFETs have to show a high stability. In particular any hysteresis in the transfer characteristic and a shift in the threshold voltage due to the applied gate bias have to be minimized to achieve highly reproducible and reliable parameters (i.e. threshold voltage, charge carrier density, on/off ratio, etc.) from a large number of samples, in order to reach a systematic and conclusive understanding of doping effects on transistor behavior.

In order to increase the stability of the doped OFETs, a thin layer of tetratetracontane (TTC) is deposited as passivation layer on the top of gate oxide as shown in Figure S1(a). TTC has been widely studied for its ability to eliminate traps, and it was shown to allow Pentacene-based OFETs to transport holes or electrons as p- or n-type transistor respectively.

The transfer characteristic of intrinsic OFETs $\left(d_{d o p}=0 \mathrm{~nm}\right)$ with varying thickness of Tetratetracontane (TTC) $(0,15,25,30 \mathrm{~nm})$ are shown in Figure S1 (b). 
As seen in Figure S1 (b) the hysteresis of OFETs is dramatically reduced from $3.1 \mathrm{~V}$ to 0.3 $V$ by increasing the thickness of TTC from $0 \mathrm{~nm}$ to $30 \mathrm{~nm}$. Meanwhile, the initially unipolar transistor characteristic (TTC thickness $=0 \mathrm{~nm}$ ) is turned into an ambipolar characteristic with increasing thickness of the TTC layer.
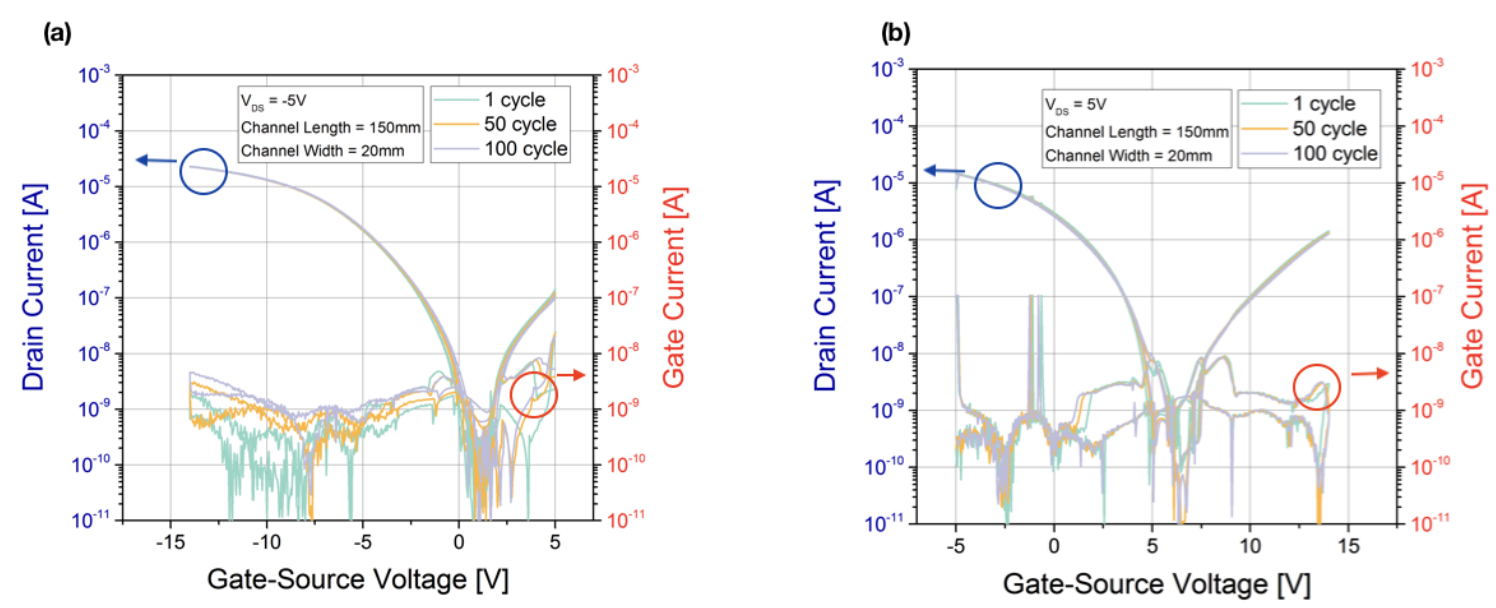

Figure S2: (a) Transfer characteristic of OFETs without doped-channels in a continuous measurement cycle, which shows a well consistency after 100 cycles of measurement: (a) p-type operation; (b) n-type operation. In order to decrease the off-state current, the drainsource voltage $\left(V_{D S}\right)$ is decreased from $\pm 15 \mathrm{~V}$ to $\pm 5 \mathrm{~V}$, which increases the on/off ratio from $10^{2}$ to $10^{5}$, measured at the transition of p- to n- type current.

Figure S2 shows the transfer characteristics of OFETs in a continuous measurement cycle up to 100 times. Given the ambipolar behavior of these OFETs, they are operated and tested as p- (Figure S2 (a)) or n-type OFETs (S2 (b)) seperately. A change in the characteristics is barely seen among the first, 50th and the 100th cycle, which indicates a good repeatability of results.

In conclusion, adding a $30 \mathrm{~nm}$ thick film of TTC to the transistor stack leads to a stable and reproducible transistor. In the remainder, all devices share an identical gate dielectric of $104 \mathrm{~nm}$ of $\mathrm{Al}_{2} \mathrm{O}_{3}$ layer and a $30 \mathrm{~nm}$ of TTC. 